\title{
Bringing Sounds into Use: Thinking of Sounds as Materials and a Sketch of Auditory Affordances
}

\author{
Christopher J. Steenson ${ }^{*}$ and Matthew W. M. Rodger
}

School of Psychology, Queen's University Belfast, United Kingdom

\begin{abstract}
We live in a richly structured auditory environment. From the sounds of cars charging towards us on the street to the sounds of music filling a dancehall, sounds like these are generally seen as being instances of things we hear but can also be understood as opportunities for action. In some circumstances, the sound of a car approaching towards us can provide critical information for the avoidance of harm. In the context of a concert venue, sociocultural practices like music can equally afford coordinated activities of movement, such as dancing or music making. Despite how evident the behavioral effects of sound are in our everyday experience, they have been sparsely accounted for within the field of psychology. Instead, most theories of auditory perception have been more concerned with understanding how sounds are passively processed and represented and how they convey information of the world, neglecting than how this information can be used for anything. Here, we argue against these previous rationalizations, suggesting instead that information is instantiated through use and, therefore, is an emergent effect of a perceiver's interaction with their environment. Drawing on theory from psychology, philosophy and anthropology, we contend that by thinking of sounds as materials, theorists and researchers alike can get to grips with the vast array of auditory affordances that we purposefully bring into use when interacting with the environment.
\end{abstract}

Keywords: Affordances, auditory perception, dance, ecological psychology, music, sensorimotor coordination.

\section{TAKING SOUNDS OUT OF THE HEAD}

Understanding the perception of sound and how we interact with auditory information is not an easy task. It is difficult to conceive an objective way of explaining auditory experiences when our perceptions of sound and our intentions surrounding these perceptions, by definition, are subjective mental phenomena. This distinction between the objective and the subjective, the mental and physical, is a problem that has dogged psychology generally over its short lifespan. Different approaches have been developed to explain these dichotomies, which have left far reaching implications on how researchers have approached more specialist areas, such as auditory perception. The dominant approaches to psychology, such as cognitive- and information processing-based approaches, explain auditory perception as the transformation of objective input (energy stimulating receptors) into subjective output (declarative descriptions of, or, responses to received input). This process of input-cognitionoutput, we argue, falls short of adequately accounting for the phenomenology of our experiences with the auditory environment.

In this paper, we instead propose an alternative view to auditory perception that can account for the various creative ways we can meaningfully use and interact with sounds. Drawing on ideas from ecological psychology, this approach takes a different form of discourse to conventional cognitive

\footnotetext{
*Address correspondence to this author at the School of Psychology, Queen's University Belfast, United Kingdom;

Tel: +44 (0) 289097 4558; E-mail: csteenson02@qub.ac.uk
}

approaches. This can be typified by its commitment to taking the meaning of sounds out of the head, and instead, placing them within the environment. We believe that in doing so, the various skilled ways we interact and coordinate our actions with the auditory environment can be truly appreciated. This distinction between sounds residing in the mind and sounds in the environment (and between representational and non-representational approaches) to psychology can be broadly defined under two different forms of discourse: a vertical or horizontal worldview $[1,2]$. In the first two sections of this paper, we aim to show how a vertical worldview has, implicitly, left us with a reductive explanation of auditory perception. Disputing this view, we outline how ecological psychology can provide an alternative, horizontal take on this phenomenon. Importantly, we also argue that ecological psychology can get closer to dealing with the difficult task of explaining how we can both meaningfully perceive and interact with sounds.

A vertical worldview $[1,2]$ is marked by an implicit treatment of objects, events and behavior as being made of discrete, underlying components. It is a view at home in scientific and philosophical modes of discourse. For example, in physics, particles and atoms are understood as the 'building blocks' from which physical objects are constructed. As such, everyday objects such as tables and chairs are seen as being made up of discrete atomistic parts that make their sum. This general way of explaining aspects of the world has led to a general approach to scientific theory that places the causes of real-world events as being within the phenomena of study, made up from discrete parts that create the surface activity being experienced. Since the beginnings of psychological science with William Wundt's structuralism, these 
reductive explanations have been a dominant discourse in the behavioral sciences as well as the physical sciences.

Within the study of behavior in psychology, the main schools of thought that have taken up this form of discourse have been the cognitive/information processing approaches. These approaches attempt to explain facets of human thought and perception by breaking the observed trait down into smaller, mechanistic, parts (much in the same way as tables are seen as being made of smaller building blocks like atoms). The 'building block' of choice for the cognitive scientist in compartmentalizing psychological phenomena has been the mental representation (e.g. [3]; see [4] for a discussion of representations). Taking perception as an example, it is generally assumed that in order to see or hear, information available to the senses must first be processed and sampled as points of sense data and (re)represented in the mind before something can be meaningfully perceived, inferred and acted upon $[5,6]$. In line with a vertical worldview, this indirect explanation of experience implies that perception and behavior are mediated by the sense-making inner-workings of the mind and therefore are caused in a way that is detached from the surface activity of the phenomena that is taking place. The meaning of a perception and the possible actions we can use it for must be dependent on cognition and the learning of appropriate responses to previously experienced stimuli $[6,7]$.

This vertical form of discourse is also evident in the field of auditory perception. In attempting to understand how a sound is perceived, research has based explanation on cognitive structures residing in the observers mind. These cognitive structures or modules are said to perform the task of indirectly processing incoming sound energy from the air medium [8]. Physical dimensions of the sound waves, such as its frequency, amplitude and duration are seen as being processed and represented as corresponding low-level perceptual dimensions of pitch, loudness and subjective duration, as well as other purported features such as timbre and the perception of space $[9,10]$. These perceptual dimensions are in turn constructed into holistic and meaningful auditory representations with the aid of past auditory experiences stored in memory [11]. In taking this stance, most research programs have focused on identifying how this transduction of physical energy, via auditory receptors, to representation in the brain occurs with the creation of things like 'auditory objects' (see [12]). The fruits of this research are a number of general psychophysical laws regarding auditory perception, such as the nonlinearities that exist for the perception of pitch as a function of sound intensity [9].

While this indirect and compartmentalized explanation of auditory perception is successful in explaining how listeners "process" low-level dimensions of sound, such as its pitch or loudness, it arguably neglects the phenomenology of auditory experience. Therefore, the holistic form of an auditory event that we meaningfully perceive has been left obscured. Indeed, focusing on the raw input of a sound (as cognitive psychologists would put it) is comparable to what Heidegger [13] referred to as listening to the "bare sound" (p. 26) of an auditory event. Heidegger argued that hearing dynamic changes in bare sensations of pitch and loudness are only perceived when one actively chooses to abstract away from a sound. Instead, Heidegger reasoned that in the immediate perception of a sound, we hear things in the world before the sensations that make them up. The two main ways of attempting to explain this have come from the (related) approaches of Gestalt and ecological psychology. The main tenant of Gestalt psychology is that perceiving the whole of a stimulus is different or "other" to the sum of its parts [14]. That is, Gestaltists argue that hearing a car is qualitatively different from the experience of any of the single frequencies from which it is constructed, leading to the idea that the additive computations performed by cognition in auditory perception should not hold. Much success has been made in applying Gestalt-grouping principles to the auditory domain, such as in the work of Bergman's auditory scene analysis [15] (see also [8]). However, these Gestalt-inspired ideas still largely fall within the discourse of a vertical worldview, with auditory gestalts and auditory streams being subject to the same status as auditory objects in ultimately being representations of the external world, which result from neural mechanisms $[16,17]$.

Developed to naturalize or "ecologize" Gestalt psychology ([18], p. 85), the writings of J. J. Gibson's ecological psychology $[19,20]$ have provided another alternative way of theorizing about auditory perception. In antithesis to representational mechanisms, ecological psychology treats perception as the direct pick-up of richly structured environmental information. This explanation bypasses the need for stimuli to be internally processed by cognition before perception is possible. With regards auditory perception particularly, it negates the need for acoustical input to be (re)organized, computed or parsed by (neuro)cognitive mechanisms (e.g. [8, 11]). Instead the theory of direct perception places meaning on how the changes in an acoustical input correspond to invariant properties of the changing environment [21-23]. This view of (auditory) perception, removes the need for explanations that hinge on mechanisms resting within the mind to do the 'hard' work of making perceptions meaningful. With the dynamics environment already providing a rich and causal structure, meaningful perceptions emerge on an equal plain between a perceiver and environment. They therefore only need to be "picked-up" or "detected", not processed or computed. As a consequence of this, the ecological approach shifts our attention away from a vertical worldview toward a horizontal one $[1,2]$ where meaningful perceptions are an emergent effect of the dynamic interactions taking place between a perceiver and their environment ( - as opposed to meaningful perceptions emerging solely within the perceiver's mind, made meaningful through an anchoring to their past experiences).

The aim of the present paper is to show how an ecological conception of auditory perception can be useful in explaining not only how we obtain information about auditory environment but also, most pertinently, how we can also act with the environment. In the next section, we will review some of the main ideas and empirical findings regarding ecological acoustics (the ecological approach to auditory perception). Despite being an invaluable body of work in curating a direct account of auditory perception, we will argue that one main caveat of ecological acoustics has been its neglect for a key aspect of Gibson's [19] landmark writings the idea of affordances (the opportunities for action made available by the environment). We will argue, in line with 
Gibson, that the meaning of an auditory perception should be defined in terms of the possible affordances that the sound offers us. The rest of the paper will involve putting forward the case of how the idea of auditory affordances can be made sense of in a way that encompasses the various types of behaviors that we engage in with the environment. We then argue that thinking of sounds as materials may be one way of accounting for this. We finish off the paper by briefly discussing some of the proposed metaphors implications in research and practice.

\section{SOUNDS IN THE WORLD}

Of the first to give full consideration to the idea of an ecological acoustics was William Gaver [21, 22]. Akin to Heidegger's [13] conception of the "bare sound", Gaver argued that the focus on sensation-like, low-level dimensions of an auditory event was an example of musical listening, where the primary focus of aural perceptions rests on the sound itself, such as focusing on the sounds changes in pitch and loudness. Like Heidegger, Gaver argued that whilst musical listening is certainly an available kind of listening we can engage in (with practice and the learning of cultural conventions; see Thinking of sounds as materials section of this paper), it is an intellectual abstraction away from how we primarily perceive sounds in their immediacy as events in the world [21,23]. Gaver argued that the most prevalent mode of listening we engage in is called everyday listening. For the listener, this mode of listening is concerned with the detection of the everyday things and happenings as they take place in the auditory environment. Essentially, this explanative turn from musical to everyday listening shifts focus away from the surface qualities of an auditory event (the bare signals of frequency, amplitude, etc.) to its structured causes. Listening is seen as an environment-orientated affair, with emphasis on how sounds can be used to inform a perceiver of the real-life things and events taking place around them. As such, when hearing an ecologically grounded auditory event we are less likely to describe it as a "pulse train of high-pitched, short duration sounds" than as "the sound of a glass breaking" [24]. In searching the literature, there are many examples of how auditory perception takes on this environment-orientated role. For example, from a young age we are able to correctly match everyday sounds to their everyday seen objects (see [25]). In adult listeners, reports and categorizations become more detailed still, with the materials, actions and the tasks that sounds are involved in all being recognized and reported [26-28].

These experimental findings into the recognition and categorization of environmental sounds lie in stark contrast to a conventional, indirect notion of auditory perception. Rather than focusing on sensation-like elements of the sound itself, these findings suggest that auditory perception is much more concerned with trying to identify how these acoustic patterns relate to environmental sources and, in particular, the causal actions that produce them [27-29]. Attempting to advance his notion of everyday listening, Gaver [21] set out to provide a preliminary framework of how this could be the case. Foremost, this framework involved turning away from an account of auditory perception that depended on the inner-processes of the mind (e.g. [11]) to an account that explained auditory perception as the result of a perceiver inter- acting with the environment. Within Gaver's account, sounds are the product of causal effects between the physical structure of objects and the unique modes of vibration they produce when excited by an external force [21, 22]. Assuming the objects structure remains untransformed, it will always reliably transfer these patterns to the air surrounding it. Thus, for a perceiver, the auditory information provided by this event will retain invariance, due to the sounds causal dependence on the structure and dynamics of the environmental objects and events that produced it. An environmental event will always generate a structured distribution of acoustic energy, which will always reliably specify that event to the perceiver [30]. Importantly, this negates the need for the mechanics of cognition to intervene by adding meaning through the use of representations (e.g. [11]), as meaning is readily present in the environments rich structure and causal dynamics. This mutuality means that auditory perception can instead be explained as being direct $[19,20]$. Consequently, it can also be argued that information does not need to be represented but rather differentiated by learning how the higher-order structure of the stimulation we receive invariantly relates to the parts of the environment we interact with $[31,32]$.

Adopting this direct account of auditory perception, many researchers have begun to investigate how listeners are able to detect environmental properties from sound. One of the principle ways of doing this has been by asking participants to identify heard objects or to discriminate between different heard object properties. For example, it has been found that participants are readily able to tell to discriminate between the differences in size of otherwise identical objects (like a ball or steel rod) based solely on the sound they produce when dropped (e.g. [33-35]). Doing so has allowed researchers to understand the specifying variables participants use to perceive aspects of auditory events. Elsewhere, research has shown that listeners can perceive the geometric shape of an object from both radiant and reverberant auditory information $[36,37]$ and that participants can also perceive the relative distance of how far away a sounding objects is from them $[38,39]$. Further still, people also seem to show keen ability to perceive the actions and attributes of other agents that may create an auditory event. Listeners have been to be able to identify a person's emotion or gender from their footsteps and state whether or not a recorded sound was produced by themselves or by another person [26, $29,40-44]$. These findings have validated the idea that listeners are able to directly pick-up the structured auditory information from how it corresponds to the invariant interactions of physical environmental forces.

\section{AUDITORY AFFORDANCES}

So, whilst cognitive explanations of auditory perception have argued our understanding of sound is dependent on the construction of mental representations, ecological acoustics has attempted to show us how the sounds we hear are already richly structured due to being a causal product of environmental processes - negating the need for representation of auditory events altogether. However, despite this large shift in theoretical explanation, cognitive and ecological investigations of auditory perception have remained starkly similar through the implementation of passive judgment style tasks 
and experiments. In striving for an understanding of "the source event and its properties" ([30], p. 80), what has been left is a theory of auditory perception that is only well equipped enough to explain how we perceive information about an event [45]. Much less understood is how our perceptions of sound can be used to guide our interactions with the environment, providing information for action [19, 45]. Essentially our argument is that whilst our perceptions need to be reliably informative of everyday events, they also need to be useful in this regard, providing information of the possible opportunities for action that are available. Indeed, these opportunities, or affordances as they are referred to, are precisely what most otherwise ecologically-driven accounts of auditory perception have left relatively implicit or ignored (e.g. [21, 22, 30]). Coined by J. J. Gibson [19], affordances are relational aspects of the environment that provide or furnish possibilities for an agent to act $[4,46]$. For example, for adult humans a staircase can afford stepping onto but for smaller children stairs may better afford climbing. In each instance, the same structured feature of the environment supports different patterns of activity in relation to the abilities of each individual, regulating their behavior in different ways. As such, affordances are dependent upon both aspects of the environment and the abilities of an individual organism (within a form of life; [47]) in order to be perceived and used $[4,46]$ (see [48] for a classic example).

Affordances are a much less discussed matter in the field auditory perception (with the exception of music, e.g. [49-52]). Of course, this does not mean that such affordances do not exist. An obvious example is hearing a car approaching from behind (see [21] for a descriptive explanation). In such circumstances, identifying the sound as one being produced by a car is important to detect, but only insofar as in how it concurrently can provide other information more critical to the dynamics of the situation. In this instance, the information of the car approaching from behind affords moving or avoiding (see further below for an extended discussion of this example). Gibson [19] argued that in order for such information to guide action effectively, it needs to be constituted relationally, pointing both ways to agent and their environment. As such, Gibson argued that the perception of information, was the perception of relationally defined affordances $[4,19,45]$. However, by defining the role perceptual information as being for the specification of the sources and properties of a sound, ecological acoustics approaches (e.g. $[21,22,30])$ have placed the meaning and understanding of auditory events as residing one-sidedly in the structure of the environment [53]. In doing so, their approaches have failed to take into account how this auditory information is formed relationally, emerging with the situated activity of the agent. As a consequence of neglecting this Gibsonian characterization of information, ecological acoustics and theories of affordances are largely ontologically incompatible. Nevertheless, we argue that affordances need to be accounted for to have a truly ecological conception of everyday listening. Specifically, to understand sounds in an everyday context, we need to also consider how they are used to control or guide our actions effectively.

Recent research is demonstrating that sounds do offer affordances for action. Take the sound of a rolling ball as one example. Research has found that listeners can perceive the speed and size of a rolling ball (to some degree of accuracy, at least) [54]. Along the lines of an ecological acoustics account, this can be explained through (and modeled by emulating) the sound-producing physics of the ball as it travels across a surface [55-57], providing structured information of the event. Recent research extends these findings, showing that the sound of a rolling ball can also offer sufficient affordances for action. When set with the task of directly or indirectly intercepting a rolling ball from its sound alone, participants are able to perform very well if not perfectly $[58,59]$. Likewise, whilst research has found that listeners can detect the size or material of an object from its resultant sound (e.g. [34, 60, 61]), this information can also be used to prospectively guide and influence aspects of listeners' reaching movements. For example, research has shown that hearing a material associated with part of an object whilst reaching for it, sways participants to grasp the part of the object that is congruent to the material they hear. [62]. Hearing other characteristics of objects such as their size has also been found to influence reaching movements. In one study conducted by Sedda, et al. [63] it was found that hearing the size of a dropped object had significant effects on the aperture of peoples grip when they were asked to reach and grasp that object. It was found that hearing a larger object caused participants to open their hand more than when they heard a smaller object [63].

As well as information produced by environmental events being important for the control of action, sounds produced by (or related to) our own actions also seem afford uses for controlling behavior. For example, research has found the sound of footsteps to be a useful cue for guiding human actions. These self-produced sounds, resulting from the interaction between feet impacting the ground during locomotion have been found to be important as reafferences for action in long-jumping, with it being found that when time-delays were introduced between foot impacts and their resultant sounds, movement performance was disrupted [64]. Other studies, such as those conducted by Young, Rodger \& Craig [44, 65] have found that hearing the recorded sounds of footsteps on gravel can provide sufficient information for participants to imitate the cadence and stride length of a model. Furthermore, it was found that these ecological sounds were able to significantly reduce variability in stride length for Parkinson's disease patients [65]. Similarly, when participants are asked to synchronize their breaths to a sound stimulus, one study has found that the ecological sounds of human breathing produce more stable breathing durations when compared to artificially synthesized sounds [66].

From the findings outlined above it seems clear that, rather than being passive receivers of information about the environment, we actively bring advantageous information into use to afford an adequate grip on the situation. Notably, when using auditory information for catching, grasping or acting in some other kind of way, how the information available corresponds to the material structure of the environment is of little concern. The latent content of a sound (i.e. its causes or "aboutness") holds no utility in and of itself [45]. Instead, what is important is how this auditory information, in relation to an agent, can provide a more or less desirable state of affairs within their present context [6]. Essentially, what is of concern is the relevant uses that the auditory in- 
formation provides [45]. Indeed - much as Tim Ingold $[2,67]$ contends in relation to the use of materials when materials in making - we argue the meaning of an auditory event does not always arise from its invariant composition or structure but through the reliable uses and functionalities it provides in relation to the user. As such, the acoustic structure produced from an environmental event is better seen as being "brought into use" ([67] p. 352) by an agent for the coordination of perception and action (within the context of the task at hand).

This idea of meaning arising with use, rather than residing in objective structure, can be demonstrated nicely with the earlier example of an approaching car. The sound of an approaching car is determined by its sound-producing mechanics, as it unfolds spatiotemporally in the environment, leading to dynamic increases in sound intensity and highfrequency components as the car moves closer ([21, 68-71]). As mentioned, these looming sounds (where a sounding object approaches a listeners point of observation) are generally associated with a undesirable state of affairs for an agent and will afford avoiding in most everyday circumstances (as generally found and inferred through anticipatory biases in judging time-to-contact/time-to-passage; e.g. $[69,71])$. This is generally attributed to the dynamics of the looming sound "specify[ing] the arrival time of the source" ([72] p. 123), allowing for an agent to act prospectively in relation to the event. However, the idea that objective dimensions of a sound, like its increasing intensity and high frequency components, hold information of arrival time - like as if predesignated with certain functionalities - seems like an erroneous attribution under the position advocated here.

By describing sounds as being "brought into use", as a relational interaction between agent and environment, the uses of a sound (i.e. the affordances it provides) should be treated as an emergent process. With situated and taskdirected activity of an agent, we argue that the same patterns of structured information the environment provides a multitude of potential uses, depending on how this information becomes available in interaction with the situated activity of the individual. This is not to deny that, in most circumstances, an approaching sound will indicate a dangerous situation and invite the appropriate response of avoidance. It is merely to point out that for other users such sounds - like the sound of a looming car - can take on other resourceful roles for agents in controlling their behavior. For example, rather than being avoided, visually impaired people report the sounds of oncoming traffic as being their most used and useful auditory cues for wayfinding in urban environments [73]. Hearing moving cars and traffic can indicate the boundary from the road to the footpath and, as such, be used to walk in a straight line and avoid veering off onto a busy street [73]. When intentionally engaged in the task of navigation, the sounds of looming traffic are brought into use due to their reliability in invariantly indicating the layout of the urban environment. Moreover, this auditory affordance is not normally perceived in sighted individuals, given their visual access to the same information. Hence, an agent's perception of (and sensitivity to) possible affordances in aspects of the environment will be driven in part by the suitability of the particular affordance to the task at hand, as well as being formed in relation to his or her individual sensitivities and skills.

\section{THINKING OF SOUNDS AS MATERIALS}

We believe the ways by which agents bring sounds purposefully into use for tasks, like auditory wayfinding, is actually much the same as the way people and animals use materials in making. This idea of thinking of sounds as materials is perfectly summed up in the way Tim Ingold [67] describes how the male weaverbird builds its nest. As Ingold puts it, the weaverbird actively explores the environment choosing materials that afford use in the immanency of the task of building. Importantly, the materials it uses, like torn grass and leaves, are adopted into the task of building because of how their structure can support and contribute to the nests evolving form. Crucial to this, in Ingold's eyes, is that the making of the nest "arises within the process of use, rather than disclosing what is, ideally if not materially, ready-made" ([67] p. 354). Analogously, as we have argued here, the affordances we detect from sound are immanent to our relevant use of them. It is through needing to navigate the urban environment that the sounds of cars emerge as an affordance for navigation, much like how through making a nest that a weaverbird discovers torn grass and leaves as useful materials for nest building. In each instance, reliable causal properties of the material environment are employed into the unfolding trajectories of each organism's journey as they interact with the environment.

Importantly, using the word material to describe a sound avoids the talk of things like "objects" or representations of some kind that convey a detached, objectified view of how sounds come "ready-made" with meaning or functionalities. Talking of materials instead places agents within the form and meaning generating process as craftsmen, who work with their perceptions to act in the world. Affordances are born out of this unfolding process of environmental interaction. Talking of a material environment $[2,32]$, in our view, emphasizes how the relationship between a using perceiver and structured environment provides a resourceful manifold of creative opportunities for action. Indeed, overall, it is a view that places discourse along a horizontal plane $[1,2]$ by focusing explanation on how the temporally extended and situated activity of the agent forms basis for behavior. Particularly, in placing the emergence of meaning as a process of interaction and controlling behavior in the environment, it is an explanation that can bypass typical vertical accounts of behavior, that see the root to meaning as residing in the mind $[1,2,6]$.

Taking the idea of sounds as materials further, we also want to point out that difference in ability, as well as being constituted by bodily capacities (like in the case of the visually impaired) or species-specific capacities (like for the weaverbird or human) are also influenced by sociocultural practices. In line with Rietveld \& Kiverstein [47], we argue that perception and use of affordances is dependent on the sociocultural practices and skills that are available within a form of life. This is important to accommodate in thinking of sounds as materials as we can adopt causal aspects of the auditory environment into many forms of skilled practice like music, dance and language. Just as craftsmen from different forms of practice may find different uses in materials, we too as perceivers find different uses available to us in sound through the types of sociocultural practices in which 
we learn and engage in. The metrical and syncopated rhythms of music generally seem to be privileged to the human form of life [74], with most people being able to synchronize their taps to a regular beat $[75,76]$ and dance to music $[77,78]$. These affordances are readily available due to their practiced uses in musical culture. However, whilst a Western listener may be able to tap out the beat of a standard 4/4 rhythm found within the normal Western conventions of music, they may not possess the skills to do so for the unfamiliar rhythms of Balkan folk music ([79]; for another skillbased example of using sound for action, see [80]). By not being familiar with the practices from which the music/sounds are a part, the relevant affordance for synchronization cannot be detected. In this way, sounds can also be considered as being "sociomaterial", as the material environment is "sculpted by our sociocultural practices into a sociomaterial environment" ([47] p. 335). As a result our abilities and skills in being able to detect and use auditory affordances are grounded in these cultural factors as well. Even in the example of auditory wayfinding [73] (see above), the affordances provided by the sounds of traffic are dependent on the normative practice of road laws by the drivers and pedestrians participating in the urban environment.

A particularly interesting example of how sound as materials can cut across traditionally separate boundaries of explanation (physical, physiological, social, and cultural) is the worksong. Worksongs have been found throughout many different work and social groups [81, 82]. Often sung as a way of collectively airing grievances and transcending the monotony of the job at hand, singing songs was also a way of coordinating members amongst a group (for an experimental example, see [83]). The general cultural conventions of rhythm and melody are brought into use, affording a collective pace or tempo to which the work could be done. Whilst debatable, some recent findings from sport psychology show music can be used as a way of coordinating action efficiently [84, 85], worksongs may have served the additional benefit of reducing energy consumption amongst the individuals of the group. Moreover though, what is most interesting to note is how these worksongs were "constituted in relation to the necessities of labour" ([82] p. 317). Far from being imposed on the working process, it seems that through the regular patterns of coordinated movement set by the task at hand, worksongs emerged as a part of the work process. Rather than the separate conventions of music and work being wedged onto one another, it seems the musical conventions available in the workers form of life potentiated the affordances of music making from the already audible and coordinated patterns of movement furnished by the labor process. For example, sailors would sing sea shanties that fitted the kind of tasks they were performing on deck - having a song for hauling ropes and setting sails and another for heaving work [82]. Other tasks, like cutting and hammering, would integrate the thump of the tools into the pulse of the song, accompanied by the grunt of the workers as they swung [81]. The sounds of hammering, for example, can be interpreted as providing a means for forming the rhythm of a song, in turn affording singing and a way of keeping time amongst the larger group that, whilst not always possible through sight or touch, is afforded through the use of sound.
With all this in mind, we feel the general sounds-asmaterials metaphor can provide a refreshing way of thinking about the matter of auditory perception. Indeed, it is a general approach that can account for how we can act with sounds in many different creative ways. This is a particularly important point as affordances have not generally been given much consideration with regard to auditory phenomena and when they have it has usually only been in relation to music (e.g. [49-52]). This approach extends the scope of auditory affordances allowing for everyday tasks, like filling a glass of water using sound [86] to dancing with music (e.g. [78]) or auditory wayfinding [73] to be mutually accounted for. Indeed, these creative and improvisatory uses of the auditory environment are poorly accounted generally for within theories of sensorimotor interaction, which have tended to focus on very constrained interactions between action and sound (such as tapping to a metronome, see below).

One way of accounting for audio-motor coordination that has become popular over the past decade has been by attributing this type of behavior to the mirror neuron system [87-89] or motor system generally (see [90]). According to these explanations, sounds are successfully perceived and interacted with by internally simulating the likely actions that produced them in the motor cortex (see [91] for a recent review in relation to music). Successful interaction from an agent therefore depends on learnt mappings between external events and internal representations of our own (and others) sound-actions effects. However, by relying on previouslylearnt models or representations of such sound-action effects, the novel and opportunistic uses of sound that emerge from agent-environment interactions are difficult to explain (see [6] for a full critique of the problem of meaning in information processing approaches). For example, a person could walk in time to a regular clapping sound, even though anatomically distinct neural regions would support the putative motor 'representation' of these events. By contrast, whilst the learning of skills and abilities do play a key role in thinking of sounds as materials, these learnt uses of environmental information are instead defined as mediating the potential forms of activity the organism can engage in rather than being the cause of that behavior per se. Therefore, the emergence of meaningful auditory affordances, in our view is a skilled agent, purposefully engaged in the task at hand, exploring and bringing into use the opportunities made available by the (socio)material environment that can be used to support his or her behavior.

\section{APPLICATIONS}

We hope that by discussing the structure of auditory events, their relation to an intentional agent and in highlighting how these relations are grounded in skilled forms of sociocultural practice, we have illustrated how the auditory environment provides a rich and resourceful manifold of action opportunities. Particularly, we believe that in appreciating how these different factors intersect in the emergence of affordances, important insights can be gained both empirically and more broadly in practice. For example, in sensorimotor synchronization research (see $[75,76]$ for extensive reviews), coordination with sensory stimuli is conventionally studied by asking participants to tap along an auditory isochronous metronome (usually consisting of a short 
noise burst or clicking sound). How people synchronize to these stimuli are well understood and have been mathematically modeled in the forms of the Wing \& Kristofferson model for continuation tapping [92] and the Vorberg and Wing model for synchronization [93]. However, within this line of study, how the sounds interact with and support the synchronization behavior of the participant is rarely accounted for and cannot be handled by models like the Vorberg and Wing model (c.f. [94]; see [95] for continuation tapping). Instead, metronomes are often treated as a way of arbitrarily demarcating time points for participants' tap onsets. Nevertheless, the dynamic structure of a metronome can have significant effects on movement performance. For example, Rodger \& Craig [96] found significant changes in the average kinematics of participants' movements when moving to continuous sounds (like a pitch glide) and discrete sounds (i.e. a metronome), with continuous sounds shown to support smoother and more harmonic movements (i.e. that are more like the smooth oscillating motion of a pendulum) than discrete, metronomic sounds [96] (see also: [97-99]). These findings give credence to the idea that by having different auditory structures available during the performance of a goal-directed task (e.g. to be perfectly in time with the metronome stimuli; [96]), different affordances will emerge in support of behavior.

For these reasons, it will be important to understand how motor-timing unfolds not only with reference to a metronome or sound event (as is often stated) but also in relation to it, with a perceiver's skills and task-directedness grounding the potential affordances that the sounds provide. Indeed, findings from judgment tasks suggest that by changing the task participants are asked to do, the auditory cues they use in informing their judgments will also change [100]. How task and context affect our use of auditory affordances in movement-based tasks is, to-date, an open question for study and one we hope to investigate in the future. Furthermore, whilst differences in skill between musicians and nonmusicians in timing behavior have been examined (see [75]), it will be interesting to see how these skills influence the selection of auditory affordances available in time-keeping tasks and how a person's audio-motor dexterity or cultural learning may transfer to other contexts detached from where they usually take place.

Other research has found that environmentally representative and action-relevant sounds, like the sound of footsteps, can also influence the control of participants' walking movements. For example, research has found that participants can reenact the walking patterns of a model by using only the sounds of footsteps on gravel [44] and that hearing our footsteps as though they are walking along different materials (like snow or concrete), whilst walking on a treadmill, can modulate subsequent measures of forward drift [101]. Applied to patients with Parkinson's disease (PD), these footstep sounds have been successfully used to encourage more healthy patterns of gait, improving the spatial variability of patients stride length and encouraging a more regular cadence in line with healthy controls when compared to the use of a simple metronome [65]. Similarly, the sound of a rolling ball has been found as useful information for PD patients in controlling their kinematics in reaching to intercept it [58]. Findings like these provide hope for new ways that sounds can be used and in the different types of tasks or contexts, like sport or movement rehabilitation. They also open up the door to new types of sound design techniques based on physical modeling of environmental sound sources that can be used for new types of tasks (e.g. [57, 102, 103]). Particularly, the sounds as materials metaphor may be useful here in guiding and understanding this design process, allowing greater refinement and development of sounds that can be implemented, by appreciating how design interacts relationally with the skills of a user $[2,47]$. In summary, thinking of sounds as materials opens up many new pathways of empirical and theoretical discussion, which will hopefully be enlightening and also useful more broadly in sound design and applied contexts like movement rehabilitation.

\section{CONCLUSION}

The argument put forward in this paper has been that to fully understand auditory perception as a meaningful process, it is not appropriate to look at sounds as auditory representations of some kind, nor is it sufficient to see the environment as transmitting or specifying meaning as a latently available part of its structure. Rather, we argue that meaning and use needs to be understood as relationally grounded process between an agent and their environment. Importantly, to appreciate these relations we need to consider the agent as skillful and intentional, as well as being sociallyand culturally-situated. The examples from different forms of practice, such as music, worksong or urban wayfinding, show that the auditory environment can afford many different possibilities for action, supporting a rich variety of behaviors (providing an agent has the ability to use them). By thinking of sounds as materials, these rich possibilities can be accounted for in a way that does not need to ontologically separate the physical (putatively 'objective') aspects of sound from the (putatively 'subjective') aspects of the agent. Furthermore, we believe that in adopting this way of thinking, research into applications of auditory guides/sonification for enhancing movement performance in different contexts, such as sport or rehabilitation, may be fruitfully advanced.

\section{CONFLICT OF INTEREST}

The authors confirm that this article content has no conflict of interest.

\section{ACKNOWLEDGEMENTS}

We would like to thank two anonymous reviewers for their helpful and insightful comments. The first author would also like to thank Nicole Andelic and Thia Sagherian Dickey for taking time to comment on and proof read earlier versions of this manuscript.

\section{REFERENCES}

[1] Van Dijk L, Withagen R. The horizontal worldview: A Wittgensteinian attitude towards scientific psychology. Theory Psychol 2014; 24: 3-18.

[2] Ingold T. Making: Anthropology, art and architecture. London: Routledge 2013.

[3] Fodor JA. The modularity of mind: an essay on faculty psychology. Cambridge, MA: MIT Press 1983

[4] Chemero A. Radical embodied cognitive science. Cambridge, MA: MIT Press 2009. 
[5] Costall AP. Are theories of perception necessary? A review of Gibson's the ecological approach to visual perception. J Exp Anal Behav 1984; 41: 109-15.

[6] Cisek P. Beyond the computer metaphor. in: núñez $r$, freeman wj, ed. reclaiming cogn primacy action. Intent Emot Exeter, UK: Imprint Academic 1999; pp. 125-42.

[7] Dewey J. The reflex arc concept in psychology. Psychol Rev 1896; 3: 357-70.

[8] Shepard R. Cognitive Psychology and Music. In: Cook PR, Ed. Music Cognation and Computrized Sound. Cambridge, MA: MIT Press, London, UK 1999; pp. 21-36.

[9] Zwicker E, Fastl H. Psychoacoustics: Facts and Models, $2^{\text {nd }}$ ed. Berling: Springer 1999.

[10] Hirsh IJ, Louis S, Watson CS. Auditory psychophysics and perception. Annu Rev Psychol 1996; 47: 461-484.

[11] McAdams S. Recognition of sound sources and events. In: McAdams S, Bigand E, Eds. Think sound Cogn Psychol Hum Audit. New York, NY: Oxford University Press 1993; pp. 146-198.

[12] Griffiths TD, Warren JD. What is an auditory object? Nat Rev Neurosci 2004; 5: 887-92.

[13] Heidegger M. Poetry, Language, Thought. New York, NY: Perenial Classics 1971.

[14] Köhler W. Gestalt Psychology. New York, NY: Liverlight Publishing Corporation 1992.

[15] Bregman A. Auditory Scene Analysis: The Perceptual Organization of Sound. Cambridge, MA: MIT Press 1994.

[16] Christison-Lagay KL, Gifford AM, Cohen YE. Neural correlates of auditory scene analysis and perception. Int J Psychophysiol 2014; 95: $238-45$.

[17] Justen C, Herbert C, Werner K, et al. Self vs. other: Neural correlates underlying agent identification based on unimodal auditory information as revealed by electrotomography (sLORETA). Neuroscience 2014; 259: 25-34.

[18] Shaw R. The Agent-Environment Interface: Simon's Indirect or Gibson's Direct Coupling? Ecol Psychol 2003; 15: 37-106.

[19] Gibson JJ. The ecological approach to visual perception. Boston, MA: Houghton Mifflin 1986.

[20] Gibson JJ. The senses considered as perceptual systems. Boston, MA: Houghton Mifflin 1966.

[21] Gaver WW. What in the World Do We Hear?: An ecological approach to auditory event perception. Ecol Psychol 1993; 5: $1-29$.

[22] Gaver WW. How do we hear in the world? explorations in ecological acoustics. Ecol Psychol 1993; 5: 285-313.

[23] Fowler C. Sound-producing sources as objects of perception: rate normalization and nonspeech perception. J Acoust Soc Am 1990; 88: 1236-49.

[24] Warren WH, Verbrugge RR. Auditory perception of breaking and bouncing events: a case study in ecological acoustics. J Exp Psychol Hum Percept Perform 1984; 10: 704-12.

[25] Jacko JA, Rosenthal DJ. Psychology of computer use: XLVI. Agerelated differences in the mapping of auditory icons to visual icons in computer interfaces for children. Percept Mot Skills 1997; 84: 1223-33.

[26] Vanderveer NJ. Ecological acoustics: Human perception of environmental sounds. Diss Abstr Int 1979; 40: 4543.

[27] Houix O, Lemaitre G, Misdariis N, et al. A lexical analysis of environmental sound categories. J Exp Psychol Appl 2012; 18: 5280 .

[28] Lemaitre G, Heller LM. Evidence for a basic level in a taxonomy of everyday action sounds. Exp Brain Res 2013; 226: 253-64.

[29] Lemaitre G, Heller LM. Auditory perception of material is fragile while action is strikingly robust. J Acoust Soc Am 2013; 131: 1337-48.

[30] Carello C, Wagman JB, Turvey MT. Acoustic Specification of Object Properties. In: Anderson J, Anderson BF, Eds. Moving image theory: Ecological considerations. Carbonadale, IL: Southern Illinois University Press 2005; pp. 79-104.

[31] Gibson EJ. Principles of perceptual learning and development. Meredihth Corporation. NY 1969.

[32] Gibson EJ. Perceptual learning in development: Some basic concepts. Ecol Psychol 2000; 12: 37-41.

[33] Lakatos S, McAdams S, Caussé R. The representation of auditory source characteristics: simple geometric form. Percept Psychophys 1997; 59: 1180-90.
[34] Grassi M, Pastore M, Lemaitre G. Looking at the world with your ears: how do we get the size of an object from its sound? Acta Psychol 2013; 143: 96-104.

[35] Carello C, Anderson KL, Kunkler-Peck AJ. Perception of object length by sound. Psychol Sci 1998; 9: 211-4.

[36] Kunkler-Peck AJ, Turvey MT. Hearing shape. J Exp Psychol Hum Percept Perform 2000; 26: 279-94.

[37] Rosenblum LD, Robart RL. Hearing silent shapes: identifying the shape of a sound-obstructing surface. Ecol Psychol 2007; 19: 35166.

[38] Rosenblum LD, Gordon MS. Echolocating distance by moving and stationary listeners. Ecol Psychol 2000; 12: 37-41.

[39] Rosenblum LD, Wuestefeld AP, Anderson KL. Auditory reachability: an affordance approach to the perception of sound source distance. Ecol Psychol 1996; 8: 1-24.

[40] Li X, Logan RJ, Pastore RE. Perception of acoustic source characteristics: Walking sounds. J Acoust Soc Am 1991; 90: 3036-49.

[41] Murgia M, Hohmann T, Galmonte A, et al. Recognising one's own motor actions through sound: The role of temporal factors. Perception 2012; 41: 976-87.

[42] Kennel C, Pizzera A, Hohmann T, et al. The perception of natural and modulated movement sounds. Perception 2014; 43: 796-804

[43] Kennel C, Hohmann T, Raab M. Action perception via auditory information: Agent identification and discrimination with complex movement sounds. J Cogn Psychol 2013; 26: 1-9.

[44] Young W, Rodger M, Craig CM. Perceiving and reenacting spatiotemporal characteristics of walking sounds. J Exp Psychol Hum Percept Perform 2013; 39: 464-76.

[45] Van Dijk L, Withagen R, Bongers RM. Information without content: A Gibsonian reply to enactivists' worries. Cognition 2015; 134: $210-4$

[46] Reed ES. Encountering the World: Toward an Ecological Psychology. Oxfor, UK: Oxford University Press 1996.

[47] Rietveld E, Kiverstein J. A rich landscape of affordances. Ecol Psychol 2014; 26: 325-52.

[48] Warren WH. Perceiving affordances: visual guidance of stair climbing. J Exp Psychol Hum Percept Perform 1984; 10: 683-703.

[49] Clarke EF. Ways of listening: an ecological approach to the perception of musical meaning. New York, NY: Oxford University Press 2005.

[50] Reybrouck M. A biosemiotic and ecological approach to music cognition: event perception between auditory listening and cognitive economy. Axiomathes 2005; 15: 229-266.

[51] Leman M. Embodied music cognition and mediation technology. Cambridge, MA: MIT Press 2008.

[52] Windsor WL, de Bezenac C. Music and affordances. Music Sci 2012; 16: 102-20.

[53] Varela F, Thompson E, Rosch E. The embodied mind. Cambridge, MT: MIT Press 1991.

[54] Houben MMJ, Kohlrausch A, Hermes DJ. Perception of the size and speed of rolling balls by sound. Speech Commun 2004; 43 : 331-45.

[55] Houben MMJ, Kohlrausch A, Hermes DJ. The contribution of spectral and temporal information to the auditory perception of the size and speed of rolling balls. Acta Acust United AC 2005; 91 : 1007-15.

[56] Rath M, Rocchesso D. Continuous Sonic Feedback from a Rolling Ball. IEEE Multimed 2005; 12: 60-9.

[57] Conan S, Derrien O, Aramaki M, et al. A synthesis model with intuitive control capabilities for rolling sounds. IEEE/ACM Trans Audio Speech Lang Process 2014; 22: 1260-73.

[58] Bieńkiewicz MMN, Young WR, Craig CM. Balls to the wall: how acoustic information from a ball in motion guides interceptive movement in people with Parkinson's disease. Neuroscience 2014; 275: 508-18.

[59] Vernat J-P, Gordon MS. Indirect interception actions by blind and sighted perceivers: the role of modality and tau. Scand J Psychol 2011; 52: 83-92.

[60] Grassi M. Do we hear size or sound? Balls dropped on plates. Percept Psychophys 2005; 67: 274-84.

[61] Rocchesso D, Fontana F. The sounding object. Mondo Estremo 2003.

[62] Castiello U, Giordano BL, Begliomini C, et al. When ears drive hands: the influence of contact sound on reaching to grasp. PLoS One 2010; 5: e12240. 
[63] Sedda A, Monaco S, Bottini G, et al. Integration of visual and auditory information for hand actions: preliminary evidence for the contribution of natural sounds to grasping. Exp Brain Res 2011; 209: 365-74.

[64] Kennel C, Streese L, Pizzera A, et al. Auditory reafferences: the influence of real-time feedback on movement control. Front Psychol 2015. doi: 10.3389/fpsyg.2015.00069

[65] Young WR, Rodger MWM, Craig CM. Auditory observation of stepping actions can cue both spatial and temporal components of gait in Parkinson's disease patients. Neuropsychologia 2014; 57C: 140-53.

[66] Murgia M, Santoro I, Tamburini G, et al. Ecological sounds affect breath duration more than artificial sounds. Psychol Res 2015. doi: 10.1007/s00426-015-0647-z

[67] Ingold T. The perception of the environment: essays in livelihood, dwelling and skill. London: Routledge 2000.

[68] Shaw B, McGowan R, Turvey M. An acoustic variable specifying time-to-contact. Ecol Psychol 1991; 3: 253-61.

[69] Neuhoff JG. An adaptive bias in the perception of looming auditory motion. Ecol Psychol 2001; 13: 87-110.

[70] Gordon MS, Rosenblum LD. Effects of intrastimulus modality change on audiovisual time-to-arrival judgments. Percept Psychophys 2005; 67: 580-94.

[71] Gordon MS, Russo F, MacDonald E. Spectral information for detection of acoustic time to arrival. Atten Percept Psychophys 2013; 75: 738-50.

[72] Neuhoff JG. Perceptual bias for rising tones. Nature 1998; 395: 123-4.

[73] Koutsoklenis A, Papadopolous K. Auditory cues used for wayfinding in urban environments by individuals with visual impairments. J Vis Impair Blind 2011; 105: 703-14.

[74] Honing H, Merchant H, Háden GP, et al. Rhesus monkeys (Macaca mulatta) detect rhythmic groups in music, but not the beat. PLoS One 2012; 7: e51369.

[75] Repp B. Sensorimotor synchronization: A review of the tapping literature. Psychon Bull Rev 2005; 12: 969-92.

[76] Repp BH, Su Y-H. Sensorimotor synchronization: a review of recent research (2006-2012). Psychon Bull Rev 2013; 20: 403-52.

[77] Toiviainen P, Luck G, Thompsom M. Embodied meter: hierarchical eigenmodes in music-induced movement. Music Percept An Interdiscip J 2010; 28: 59-70.

[78] Burger B, Thompson MR, Luck G, et al. Influences of rhythm- and timbre-related musical features on characteristics of music-induced movement. Front Psychol 2013; 4: 183.

[79] Snyder JS, Hannon EE, Large EW, et al. Synchronization and continuation tapping to complex meters. Music Percept 2006; 24: 135-46.

[80] Cesari P, Camponogara I, Papetti S, et al. Might as well jump: sound affects muscle activation in skateboarding. PLoS One 2014; 9: e90156.

[81] Brown S. Negro folk expression: spirituals, seculars, ballads and work songs. Phylon 1953; 14: 45-61.

[82] Korczynki M. Music at work: a historical overview towards. Folk Music J 2003; 8: 314-34

[83] Demos AP, Chaffin R, Begosh KT, et al. Rocking to the beat: effects of music and partner's movements on spontaneous interpersonal coordination. J Exp Psychol Gen 2012; 141: 49-53.
[84] Karageorghis CI, Priest D-L. Music in the exercise domain: a review and synthesis (Part I). Int Rev Sport Exerc Psychol 2012; 5: 44-66.

[85] Karageorghis CI, Priest D-L. Music in the exercise domain: a review and synthesis (Part II). Int Rev Sport Exerc Psychol 2012; 5: 67-84.

[86] Cabe P, Pittenger JB. Human sensitivity to acoustic information from vessel filling. J Exp Psychol Hum Percept Perform 2000; 26: 313-24.

[87] Kohler E, Keysers C, Umiltà MA, et al. Hearing sounds, understanding actions: action representation in mirror neurons. Science 2002; 297: 846-8.

[88] Zatorre RJ, Chen JL, Penhune VB. When the brain plays music: auditory-motor interactions in music perception and production. Nat Rev Neurosci 2007; 8: 547-58.

[89] Chen J, Penhune V, Zatorre R. Listening to musical rhythms recruits motor regions of the brain. Cereb cortex 2008; 18: 284454.

[90] Schubotz RI. Prediction of external events with our motor system: towards a new framework. Trends Cogn Sci 2007; 11: 211-8.

[91] Novembre G, Keller PE. A conceptual review on action-perception coupling in the musicians' brain: what is it good for? Front Hum Neurosci 2014; 8: 1-11.

[92] Wing AM, Kristofferson AB. Response delays and the timing of discrete motor responses. Percept Psychophys 1973; 14: 5-12.

[93] Vorberg D, Wing A. Modeling variability and dependence in timing. In: Heuer H, Keele SW, Eds. Handbook of perception and action Vol. 3. motor skills London, UK: Academic Press; pp. 181262.

[94] Bieńkiewicz MMN, Rodger MWM, Craig CM. Timekeeping strategies operate independently from spatial and accuracy demands in beat-interception movements. Exp Brain Res 2012; 222: 241-53.

[95] Ammirante P, Thompson WF. Continuation tapping to triggered melodies: motor resonance effects of melodic motion. Exp Brain Res 2012; 216: 51-60.

[96] Rodger MWM, Craig CM. Timing movements to interval durations specified by discrete or continuous sounds. Exp Brain Res 2011; 214: 393-402.

[97] Varlet M, Marin L, Issartel J, et al. Continuity of visual and auditory rhythms influences sensorimotor coordination. PLoS One 2012; 7: e44082.

[98] Hove M, Keller P, Krumhansl C. Sensorimotor synchronization with chords containing tone-onset asynchronies. Percept Psychophys 2007; 69: 699-708.

[99] McAnally KI. Timing of finger tapping to frequency modulated acoustic stimuli. Acta Psychol 2002; 109: 331-8.

[100] McAdams S, Roussarie V, Chaigne A, et al. The psychomechanics of simulated sound sources: material properties of impacted thin plates. J Acoust Soc Am 2010; 128: 1401-13.

[101] Turchet L, Camponogara I, Cesari P. Interactive footstep sounds modulate the perceptual-motor aftereffect of treadmill walking. Exp Brain Res 2015; 233(1): 205-14.

[102] Rodger MWM, Young WR, Craig CM. Synthesis of walking sounds for alleviating gait disturbances in Parkinson's disease. IEEE Trans Neural Syst Rehabil Eng 2014; 22: 543-8.

[103] Conan S, Thoret E, Aramaki M, et al. An intuitive synthesiser of continuous-interaction sounds: rubbing, scratching, and rolling. Comput Music J 2014; 38: 24-37.

This is an open access articles licensed under the terms of the Creative Commons Attribution-Non-Commercial 4.0 International Public License (CC BY-NC 4.0) (https://creativecommons.org/licenses/by-nc/4.0/legalcode), which permits unrestricted, non-commercial use, distribution and reproduction in any medium, provided that the work is properly cited. 